\title{
Isolation of Enterococcus from Various Clinical Samples and Their Antimicrobial Susceptibility Pattern in a Tertiary Care Hospital
}

\author{
M. Paul*, P.S. Nirwan and P. Srivastava \\ Department of Microbiology, National Institute of Medical Science and Research, Jaipur, \\ Rajasthan, 303121(India) \\ *Corresponding author
}

\section{A B S T R A C T}

\begin{tabular}{|l|}
\hline Ke y w o r d s \\
$\begin{array}{l}\text { Enterococci, } \\
\text { High level } \\
\text { aminoglycoside } \\
\text { resistance }\end{array}$ \\
\hline Article Info \\
\hline $\begin{array}{l}\text { Accepted: } \\
\text { 22 January } 2017 \\
\text { Available Online: } \\
\text { 10 February } 2017\end{array}$ \\
\hline
\end{tabular}

The emergence of Enterococcus species in causing nosocomial infections poses a therapeutic challenge to clinicians. Enterococci are intrinsically resistance to multiple antibiotics. Acquired resistance to commonly used antibiotics like Ampicillin, Vancomycin and Aminoglycosides have made the situation worse and difficult to treat serious enterococcal infections. The present study aimed to isolate Enterococcus from various clinical samples and their antimicrobial susceptibility pattern in a tertiary care hospital. A total of 102 Enterococcus species were isolated from various clinical samples were identified by various conventional biochemical methods. Antimicrobial susceptibility was detected by Kirby Bauer disc diffusion method as per CLSI guidelines. A total 102 Enterococcus species isolated from various clinical samples in which 81 were E. faecalis, 18 were E. faecium and 3 were other Enterococcus. Their antibiotic susceptibility pattern is E. faecium show more resistance than E. faecalis. We hereby conclude that Enterococcus isolated from various clinical samples must be routinely screened for various drugs to prevent drug resistance in hospital settings for serious Enterococcal infections.

\section{Introduction}

In 1899, France Thiercelin had first used the name "Enterocoque" in a published paper (Theircelin et al., 1899). The term Enterococcus derived from their presence in the intestinal tract as a normal flora. Enterococci are gram positive bacteria that typically appear as a pair of oval cocci, the cell are arranged at an angle to each other. The term Enterococcus was used for organism that grows at $10^{\circ} \mathrm{C}$ and $45^{\circ} \mathrm{C}$, in $6.5 \% \mathrm{NaCl}$, and at $\mathrm{pH} 9.6$ and which survived $60^{\circ} \mathrm{C}$ for $30 \mathrm{~min}$. They are normal resident of gastrointestinal and billiary tracts and in lower numbers in the vagina and male urethra. However when they colonize where they are not normally found they may become pathogen. They are becoming increasingly important agent of human disease, largely because of their resistance to antimicrobial agents. Among several species which belong to genus Enterococcus, E. faecalis the most common isolate, have association with 80$90 \%$ of human Enterococcal infection. E. faecium isolated from $10-15 \%$ of infections (Washington). Other Enterococcal species like E. malodoratous, E. avium, E.cecorum, E. gallinarum, E. raffinosus, E. casseliflavus, E. dispar, E. hirae, E. durans, and E. mundtii are infrequently isolated from human infections. Enterococci being 2nd most 
common cause of nosocomial urinary tract infection and wound infection and 3rd common cause of nosocomial bacteraemias are Enterococcus (Moellering, 1992). They have emerged an important nosocomial agent due to their colonizing ability and multidrug resistance (Antalek et al., 1995; Buschelman et al., 1993).

They exhibit resistance to multiple commonly used antibiotics like aminoglycoside and cephalosporins because of their ability to attain and transfer the resistance genes giving rise to resistance to high level aminoglycosides and glycopeptides. Such resistance could be treated with ampicillin or vancomycin with or without aminoglycoside or teicoplanin. High level aminoglycoside resistance HLAR (MIC>2000 microgram / $\mathrm{ml})$ has emerged recently among enterococci, it may be ribosomally mediated or because of production of inactivated enzymes. The limited choice of efficient therapy in serious Enterococcal infection has been complicated due to resistance to ampicillin, high level aminoglycoside and glycopeptides. This poses therapeutic challenges to physician. Enterococcal infection like bacteraemia and endocarditis needs treatment with combination of antibiotics which includes penicillin group of drugs like ampicillin and penicillin $\mathrm{G}$ susceptible to Enterococcus species are susceptible and an aminoglycoside like gentamicin and Streptomycin for which Enterococcus isolates do not show high level resistance. But this would also be a therapeutic failure, if the isolate is HLAR. In such cases other antibiotics like vancomycin, linezolid, teicoplanin, quinpristin/ dalfopristin, etc may be useful depending on sensitivity profile.

\section{Materials and Methods}

The study was conducted in the hospital of National Institute of Medical Science and
Research, Jaipur. And was done on various clinical samples of IPD and OPD patients attending NIMS hospital Jaipur during the period of January 2015 to June 2016. The study population includes the patient of all age group and samples collected as per standard guidelines only. Various clinical samples like urine, blood, pus, stool, wound swab, sputum, body fluids, etc were collected by all aseptic technique in sterile container. Then they were inoculated on Blood agar, MacConkey agar and Nutrient agar and incubated at $37^{\circ} \mathrm{C}$ for $18-24 \mathrm{hrs}$.

On Blood agar circular, translucent, smooth, convex colonies of $1-2 \mathrm{~mm}$ in diameter, with regular margins showing either alpha or nonhemolytic colonies. On MacConkey agar they form small, $0.5-1 \mathrm{~mm}$ magenta coloured colonies. After that colony morphology is observed and processed further. Identification is done on the basis of Gram staining and biochemical reactions as per standard protocol like catalase test, bile esculin test, PYR test, growth at $45^{\circ} \mathrm{C}$, salt tolerance test $6.5 \%$, growth at alkaline $\mathrm{pH}$ 9.6, arginine dihydrolase test, hippurate hydrolysis test, potassium tellurite reduction test, sugar fermentation test.

Antibiotic sensitivity testing was done using Kirby-Baeur disc diffusion method as per CLSI guidelines. The antibiotics disc used are ampicillin $10 \mu \mathrm{g}$, nitrofurantoin $300 \mu \mathrm{g}$,

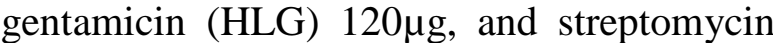
(HLS) $300 \mu \mathrm{g}$, ciprofloxacin $5 \mu \mathrm{g}$, vancomycin

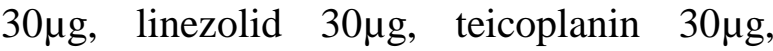

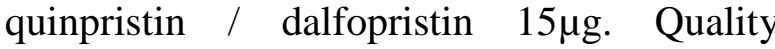
controlled used was E. faecalis ATCC 29212.

\section{Results and Discussion}

Maximum number of patients are in age group 51-60 years i.e. 18 (17.7\%) followed by 61-70 years i.e. 17 (16.7\%), 21-30 years i.e. $15(14.7 \%), 31-40$ and <10years i.e. 14 
(13.7\%) each, 41-50years i.e.13 (12.8\%), 1120 years i.e. $8(7.8 \%)$ and least from age above 70years i.e. 3 (2.9\%) (Fig. 1).

Maximum samples from which Enterococcus was isolated is urine i.e. $73(71.5 \%)$, followed by blood and pus i.e. $12(11.9 \%)$ and 10 $(9.9 \%)$ respectively.

Maximum patients are from IPD i.e. 74 (72.5\%) and OPD i.e. 28 (27.5\%) (Fig. 2).

Maximum isolate is E. faecalis i.e. 81 (79.4\%) followed by E. faecium i.e. 18 (17.7\%) and other Enterococcus i.e. 3 (2.9\%).

Table 2 shows distribution according to susceptibility and resistance pattern of different drugs. The susceptibility and resistance pattern of drugs used in the study was depicted, in which vancomycin, linezolid and teicoplanin shows $100 \%$ susceptibility. ciprofloxacin, ampicillin, quinpristindalfopristin, nitrofurantoin, high level gentamicin and Streptomycin shows $71.5 \%$, $21.5 \%, 10.7 \%, 82.1 \%, 55.8 \%$ and $50 \%$ susceptibility and $28.4 \%, 78.4 \%$ and $89.2 \%$. $16.4 \%, \quad 44.1 \%$ and $50 \%$ resistance respectively. The findings were found to be statistically significant.

During recent year, there is increased interest in Enterococci because of their ability to cause serious infection and their increasing resistance of many antimicrobials. In the present study 102 Enterococcus were isolated from 1200 various clinical samples like urine, pus, blood, wound swab, Foley's tip, Endotracheal tip from patients in OPD, Wards and ICU's (Table 1 and Fig. 3). Bacterial isolates were identified and speciated based on colony characters, morphology on gram staining, biochemical reactions, using conventional test scheme by Facklam and Collins (1989). Antimicrobial susceptibility was done by Kirby Baeur disc diffusion method.

In the present study most of the patients were from age group 51-60years i.e. 17.7\% Which is comparable to the study of Palaniswamy et al., (2013) and Sivasankari et al., (2013) whereas in another study by Telkar et al., (2012) showed maximum patients from age group 0-20yrs and Bose et al., (2012) showed most patients from 21-30 years which is slightly lower age group from present study. Majority of patients were males $53.5 \%$ in the study compared to females $45.7 \%$ with a male female ratio of 1.17:1. Most of the male patients belong to age group of 51-60 years $(10.8 \%)$ and female in the age group of 21-30 years and $31-40$ years $(8.8 \%)$ years which is comparable to study of Telkar Anjana et al., (2012) and Golia et al., (2014), whereas Puneet et al., (2014) showed more female to male ratio.

Table.1 Distribution of Enterococcal isolates from different clinical samples

\begin{tabular}{|l|l|l|}
\hline Samples & No. & $($ \%) \\
\hline Urine & 73 & 71.5 \\
\hline Blood & 12 & 11.9 \\
\hline Pus & 10 & 9.9 \\
\hline Wound swab & 3 & 2.9 \\
\hline Foley's tip & 3 & 2.9 \\
\hline Endotracheal tube tip & 1 & 0.9 \\
\hline Total & $\mathbf{1 0 2}$ & $\mathbf{1 0 0 . 0}$ \\
\hline
\end{tabular}


Table.2 Distribution according to Susceptibility and Resistance pattern of different drugs

\begin{tabular}{|l|c|c|c|c|}
\hline \multirow{2}{*}{ Drugs } & \multicolumn{2}{c|}{ Susceptibility } & \multicolumn{2}{c|}{ Resistance } \\
\cline { 2 - 5 } & No. & $\mathbf{( \% )}$ & No. & $\mathbf{( \% )}$ \\
\hline Vancomycin (n=102) & 102 & 100 & 0 & 0 \\
\hline Linezolid (n=102) & 102 & 100 & 0 & 0 \\
\hline Teicoplanin (n=102) & 102 & 100 & 0 & 0 \\
\hline Ciprofloxacin (n=102) & 73 & 71.5 & 29 & 28.4 \\
\hline Ampicillin (n=102) & 22 & 21.5 & 80 & 78.4 \\
\hline Quinpristin-Dalfopristin (n=102) & 11 & 10.7 & 91 & 89.2 \\
\hline Nitrofurantoin (n=73) & 60 & 82.1 & 12 & 16.4 \\
\hline High level Gentamicin $(\mathrm{n}=102)$ & 57 & 55.8 & 45 & 44.1 \\
\hline High level Streptomycin $(\mathrm{n}=102)$ & 51 & 50 & 51 & 50 \\
\hline
\end{tabular}

$\mathrm{X}^{2}=138.1572 \mathrm{P}<0.00001 \mathrm{P}<0.05$ significant

Fig.1 Distribution of patients according to age

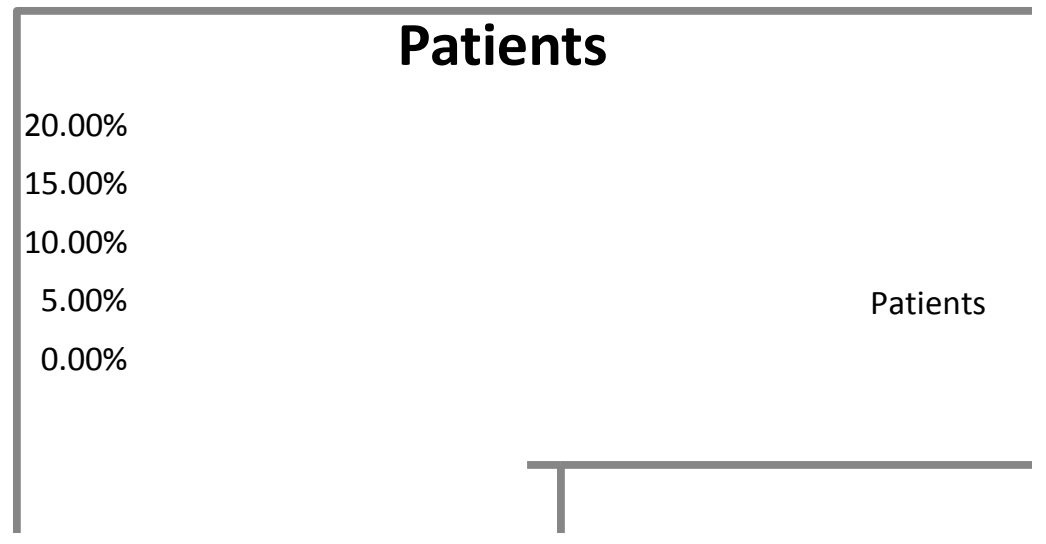

Fig.2 Distribution of patients according to OPD and IPD

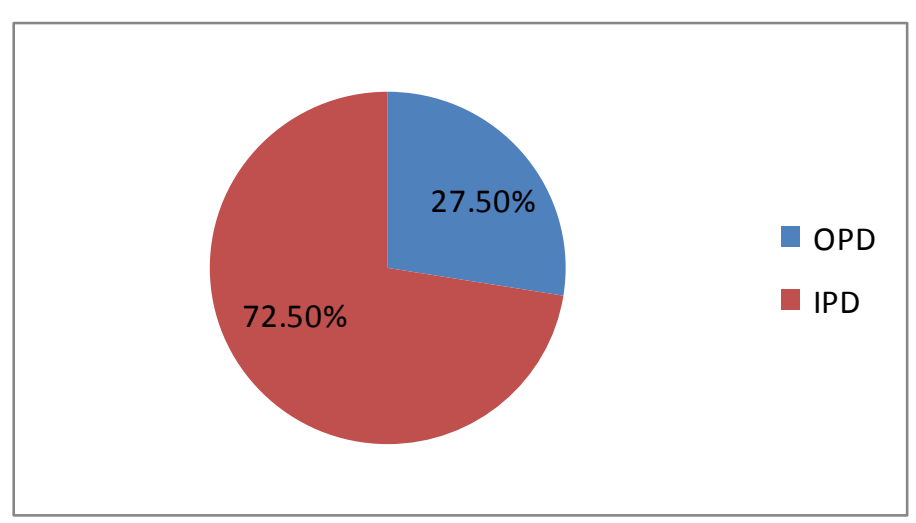


Fig.3 Distribution of E. faecalis, E. faecium and other Enterococci from various clinical samples

$\begin{array}{lll} & \mathbf{\%} & \\ & & \\ & & \\ 17.90 \% & & \\ & 79.40 \% & \text { E. faecalis } \\ & & \text { E. faecium } \\ & & \text { Other } \\ & \\ & \\ & \end{array}$

Most of the samples in study from which Enterococcus isolated is urine $71.5 \%$ followed by blood $11.9 \%$, pus $9.9 \%$, others like wound swab $2.9 \%$, Foley's tip $2.9 \%$ and Endotracheal tip $0.9 \%$. Similar results were shown by other authors. Mittal et al., (2016) Lall et al., (2014) Suresh et al., (2013) whereas Golia et al., (2014) reported maximum samples from urine, followed by pus, blood, others, which is slightly different from present study, Sreeja et al., (2012) reported maximum samples blood $58 \%$ followed by pus i.e. $43 \%$ and urine $31 \%$ respectively different from our study. Maximum patients are from wards $72.5 \%$ followed by ICUs $28.4 \%$ and OPD $27.5 \%$. Similar to the study done by Mittal et al., (2016), Lall et al., (2014), Agarwal et al., $79.4 \%$ E. faecalis, $17.7 \%$ E. faecium and $2.9 \%$ other Enterococcus was isolated in this study. Nearly similar results were obtained by different authors. Gangurde et al., (2014), Mulla et al., (2012), Adhikari (2010), whereas Lall et al., (2014), Deshpande et al., (2013), Mendiratta et al., (2008) isolated only two species in their study.

In present study vancomycin, linezolid and teicoplanin shows $100 \%$ susceptibility by disc diffusion method. Similar to the study of Suresh et al., (2013), Lall et al., (2014) whereas in the study performed by Mulla et al., shows $100 \%$ sensitivity of linezolid and Teicoplanin whereas vancomycin is only $86 \%$ sensitive and in study of Puneet et al., (2014) linezolid is $100 \%$ sensitive whereas vancomycin and Teicoplanin are $86 \%$ sensitive each. Ampicillin, ciprofloxacin, quinpristin-dalfopristin (pristinomycin) and nitrofurantoin shows $78.4 \% 28.4 \%, 89.2 \%$ and $16.4 \%$ resistance respectively similar to study of Lall et al., (2014) whereas Suresh et al., (2013) in his study reported 54\% resistance each in ampicillin and ciprofloxacin and nitrofurantoin 100\% sensitive and Puneet et al., (2014) showed $95 \%$ and $62 \%$ resistance in ampicillin and ciprofloxacin respectively which slightly higher than present study with nitrofurantoin $100 \%$ sensitive. Out of 102 Enterococcus isolated $44.1 \%$ were HLGR and $50 \%$ were HLSR, $49.3 \%$ and $46.9 \%$ strains of $E$. faecalis are HLGR and HLSR respectively and $94.4 \%$ and $72.2 \%$ are HLGR and HLSR of E. faecium respectively. Similar results were shown by Puneet et al., (2014), Adhikari (2010) and Lall et al., (2014). Hence it is concluded that Enterococci being the common cause of hospital acquired infections and bacteraemias with their increasing resistance to multiple drugs, the treatment has become a challenge for the physician. So it is 
important to know the susceptibility pattern of the organism and routine screening should be done in patients suffering from Enterococcal infections as it will support appropriate treatment strategies in cases of Enterococcal infection particularly life threatening infection and will help the clinician in treating such patients and in minimizing the speed of antibiotic resistance in the community and in the hospital.

\section{Acknowledgement}

I would like to thanks my department and my teachers for their constant guidance and help.

\section{References}

Adhikari Luna. 2010. High Level Aminoglycoside resistance and reduced susceptibility to Vancomycin in Nosocomial Enterococci. J. Glob. Infect. Dis., Vol 2issue 3, 231-235.

Agarwal Jyotsana, Kalyan Rajkumar, Singh Mastan. 2009. High level aminoglycoside resistance and $\beta$ lactamase production in Enterococci at a tertiary care hospital in India. Jpnj. J. Infect. Dis., 62: 158-159.

Antalek, M.D., Mylotte, J.M., Lesse, A.J., et al., 1995. Clinical and molecular epidemiology of Enterococcus bacteraemias with special reference to strains with high level resistance to Gentamicin. Clin. Infect. Dis., 20: 103109.

Bhatt Maj Puneet, Patel Anubha, Sahni Brig A.K. et al., 2014. Emergence of Multidrug resistance Enterococci at a tertiary care centre. Med. J. Armed Forces India, 139-144.

Bose, S., Ghosh Atindra Krishna, Barapatre Rekha. 2012. Prevalence of drug resistance among Enterococcus spp. From a tertiary care hospital. Int. J. med. health sci., Vol1 issue 3, 38-44.
Buschelman, B.J., Bale, M.J., Jones, R.N. 1985. Species identification and determination of high level aminoglycoside resistance among Enterococci; comparison study of sterile body fluids isolates; 1985-1991, diagn Microbiol. Infect dis., 16: 119-122.

Deshpande, R., Vaibhav, Karmarkar, G., Mohan, Mehta, R., Preeti. 2013. Prevalence of multidrug resistance Enterococci in a tertiary acre hospital in Mumbai, India. J. Infect. Dev. Ctries., Vol 7 issue 2, 155-158.

Facklam, R.R., Collins, M.D. 1989. Identification of Enterococcus species isolated from human infections by a conventional test scheme. J. Clin. Microbiol., 27(4): 731-4.

Gangurde Nita, Mane Manisha, Phatale Sunita. 2014. Prevalence of multidrug resistant Enterococci in a tertiary care hospital in India: A growing threat. Open J. Med. Microbiol., 4: 11-15.

Golia Saroj, A.R. Nirmala, S. Kamath, B. Asha. 2014. Isolation and Speciation of Enterococci from various clinical samples and their antimicrobial susceptibility pattern with special reference to High Level Aminoglycoside resistance. Int. J Med Res Health Sci., Vol 03 Issue 03, 2014; 526-529.

Lall Niharika, Basak Shilpi. 2014. High Level Aminoglycoside resistant Enterococcus species: A Study. Int. J. Cur. Res. Rev., Vol 06 issue 03, 16-21.

Mendiratta, D.K., Kaur, H., Deotale, V., et al., 2008. Status of High Level Aminoglycoside resistance Enterococcus faecium and Enterococcus faecalis in a rural hospital of central India. Indian J. Med. Microbiol., 26(4): 369-71.

Mittal Seema, Singla Pooja, Deep Antariksha, et al., 2016. Vancomycin and High Level Aminoglycoside Resistance in 
Enterococcus spp. In a Tertiary Health Care Centre: A Therapeutic Concern. Hindawi Publishing Corporation, $J$. Pathogens, Volume, Article ID 8262561; $1-5$.

Moellering, R.C. Jr. 1992. Emergence of Enterococcus as a significant pathogen. Clin. Infect. Dis., 14: 1173-1178.

Mulla Summaiya, Patel Kinjal, G., Panwala Tanvi. et al., 2012. Prevalence of Enterococci with higher resistance level in a tertiary care hospital: A matter of concern. Research gate. Vol 2 issue 1, 111.

Palaniswamy Sraswathy, Karunakaran Sankari, Narayan Shankara. Antimicrobial resistance profile and characterization of Enterococcus species from various clinical samples in a tertiary care hospital. Int $\mathrm{J}$ Med Research and health sciences. Vol 2 issue 32013 ; 328-332.

Sivasankari, S., V.M. Somasunder, S. Senthamarai. et al., 2013. Detection of High Level Resistant Enterococci in a tertiary care hospital. IOSR J. Pharmacy and Biol. Sci., Vol 8 issue 5. 2013; 5357.

Sreeja, S., Babu, P.R. Sreenivasa, Prathab, A.G. The prevalence and the characterization of the Enterococcus species from various clinical samples in a tertiary care hospital. JCDR, Vol 6 issue 9, 1486-88.

Suresh, K., Saripriya, B., Viswanath, G. 2013. Isolation, speciation and determination of High Level Aminoglycoside resistance of Enterococci among Hospitalized patients in Davangere. NJLM, vol 2 issue 1, 12-15.

Telkar Anjana, Baragundi, C., Mahesh, et al., 2012. Change in the prevalence and antibiotic resistance of the Enterococcal species isolated from Blood cultures. $J C D R$, Vol 6 issue 3, 405-408.

Theircelin, M.E.S. 1899. Run diplococque saprophyte de I'intestine susceptible de devenir pathogen. CR Soc. Biol., 5: 26971.

Washington Winn, Jr., Strephen Auen, William Jarda, et al., Koneman's color atlas and Textbook of Diagnostic Microbiology, $6^{\text {th }}$ ed. Philadelphia: Lippincott Williams and Wilkins;2006. Chapter 13, Gram positive cocci part II: Streptococci, Enterococci and the "Streptococcus-like" bacteria; p. 672674

\section{How to cite this article:}

Paul, M., P.S Nirwan and Srivastava, P. 2017. Isolation of Enterococcus from Various Clinical Samples and Their Antimicrobial Susceptibility Pattern in a Tertiary Care Hospital. Int.J.Curr.Microbiol.App.Sci. 6(2): 1326-1332.

doi: http://dx.doi.org/10.20546/ijcmas.2017.602.150 